\title{
How to visualize and reconstruct the pulmonary valve and contiguous structures with intracardiac echocardiography: a simplified multi-step approach.
}

\author{
Francesco De Sensi ${ }^{1}$, Luigi Addonisio ${ }^{2}$, Gennaro Miracapillo ${ }^{2}$, Marco Breschi $^{2}$, Paolo \\ Orselli $^{2}$, Alberto Cresti ${ }^{1}$, and Ugo Limbruno ${ }^{2}$ \\ ${ }^{1}$ usl 9 \\ ${ }^{2}$ Misericordia Hospital
}

September 21, 2020

\begin{abstract}
Ventricular tachycardia and premature ventricular complexes (PVCs) arising from right ventricular outflow tract (RVOT) are the most common type of ventricular arrhythmias (VAs) in patients without structural heart disease. Radiofrequency ablation is now the gold standard of treatment in this setting due to high efficacy rates and optimal safety profile [2] During the last few years, the pulmonary valve (PV) and the pulmonary artery (PA) have attracted much attention as reliable sites of origin of RVOT-type arrhythmias. In the mean while intracardiac echocardiogram (ICE) has undoubtedly improved our understanding and approach to manage these arrhythmias accurately characterizing the PV and its contiguous structures. Aim of this paper is to provide an illustrated step-by-step guide on how to use ICE with the CARTOSOUND? module to visualize and reconstruct 3D shell of the RV, the PV, as well of other anatomical structures (i.e., the aortic valve and coronary arteries) to perform aware and safe ablation in this region. A new reconsideration of the existent classification of these VAs is also provided.
\end{abstract}

\section{Keywords}

Intracardiac echocardiography, pulmonary valve PVCs, outflow tract ventricular arrhythmias

Main text (words count: 2935)

Ventricular tachycardia and premature ventricular complexes (PVCs) arising from right ventricular outflow tract (RVOT) are the most common type of ventricular arrhythmias (VAs) in patients without structural heart disease [1]. Radiofrequency ablation is now the gold standard of treatment in this setting due to high efficacy rates and optimal safety profile [2]. Using fluoroscopy and then electro-anatomical mapping, in order to standardize mapping and ablation procedures, RVOT was conventionally divided into a "septal" wall, postero-medially oriented and facing the aortic root and the proximal part of aorta, and the anterolaterally directed free wall. Each one was therefore divided in three equal areas, named 1 posterior, 2 median and 3 anterior [3]. RVOT arrhythmias more commonly originate and could be ablated from the septal areas than from free wall [4]. During the last few years, the pulmonary valve (PV) and the pulmonary artery (PA) have attracted much attention as reliable sites of origin of RVOT-type arrhythmias. Since seminal reports by Timmermans et al, several series of left bundle branch block (LBBB) type VAs were described originating from or above the pulmonary valve $[5,6]$. Distinct EKG features may rise suspicion of PVCs originating from PV or PA, larger R/S ratio in V2, late ([?] V4) precordial transition, tall $\mathrm{R}$ waves in the inferior leads, predominantly negative forces in $\mathrm{I}, \mathrm{aVL} / \mathrm{aVR}$ ratio of $\mathrm{Q}$ wave amplitude $>1$ [7-9]. In order to acquire better contact with the cusps above the PV and on the PA wall the ablator catheter can be advanced and deflected in a "candy cane" shape (reversed U-curve technique) [10-12]. Interestingly, mapping in these regions 
reveals a late sharp potential in the sinus beats which inverses becoming pre-potential during PVCs. In a recent experience, Zhang and colleagues performed radiofrequency ablation of RVOT-type VAs based on the mapping and ablation of pulmonary sinus cusps (PSCs) as the first-line strategy, eliminating 90\% (81/90) of arrhythmias [13]. According to this observation it could be speculated that PV represents the "real" source of the ectopy, historically considered to originate from regions below the valve, and ablation in ROVT is efficacy "simply" when disconnects the focus from the rest of myocardium. Interesting histopathological observations support this theory by showing that ventricular myocardium may extend into the PA beyond the semilunar valve. These myocardial sleeves, which are as long as those located in the pulmonary veins and enhancing atrial fibrillation, are characterized by abnormal automaticity and/or triggered activity, thus capable of firing the ventricular ectopy [14,15]. Myocardial voltage extension into the pulmonary artery in humans has been also demonstrated in vivo using a three-dimensional (3D) mapping system (CARTO?, Johnson \& Johnson, Biosense Webster) integrated with intracardiac echocardiography (ICE) scans (CARTOSOUND? module) to accurately localize the PV plane. Near field, high voltage electrograms were recorded in 11 out of 24 patients (46\%) with an ablation catheter above the, ICE localized, PV. In all patients, arrhythmic foci were localized in the PA (median $8.2 \mathrm{~mm}$ above the PV) and reclassified as pulmonary arterial arrhythmias [16]. This example shows how ICE has undoubtedly improved our understanding and approach to manage these arrhythmias and how accurate and detailed characterization of the PV and its contiguous structures is crucial for mapping, ablating, and better reclassifying the origin of these types of VAs. Aim of this paper is to provide an illustrated step-by-step guide on how to use ICE with the CARTOSOUND? module to visualize and reconstruct 3D shell of the RV, the PV, as well of other anatomical structures (i.e., the aortic valve and coronary arteries) to perform aware and safe ablation in this region.

\section{Anatomical considerations}

Anatomically, the PV is formed by three semilunar cusps attached to connective tissue of the annulus, a ring-shaped structure, with a mean diameter of $20 \mathrm{~mm}$, that delimits the right ventricle chamber at the junction with the pulmonary arterial trunk. PV consists of three cusps: anterior, left, and right, separated by a commissure with an overlap area between cusps called the lunula. On the superior border of the PV, there is a pocket formed by the valve cusp and adjacent arterial wall, called the sinus of Valsalva [17]. The left cusp is the closest to the aortic root, the aortic valve, and the epicardial aspect of basal left ventricle, being these structures located posteriorly to it (Fig. 1). Normally, left cusp is close to the left main coronary artery and to the proximal part of anterior interventricular descending branch, before it approaches the commissure between the left and anterior cusp and the medial side of it [18]. This close proximity has been exploited to ablate, from the left pulmonic cusp, arrhythmias originating from the left ventricular summit [19]. The remaining part of the anterior cusp and the right cusp are considered non-septal ones because they lie above anterolateral free wall, in contact with the right atrial appendage. In normal hearts, the right coronary artery originates from the right sinus of Valsalva beside the right ventricle at a level that is far below the valve, and separates from RVOT as it then goes down through the atrioventricular groove. An important aspect to keep in mind when dealing with PV reconstruction is that the valve lies on an oblique transverse plan, with the left cusp tilted more downward compared to the other cusps [20]. Furthermore, although the semilunar valves are approximately on the same level, the pulmonary valve is oriented on a perpendicular plane with respect to the aortic valve. This explains why the aortic valve is displayed on face during intracardiac echo visualization (2D), while the pulmonary valve is seen on edge, and vice-versa [21]. Both short- and long-axis views need to be visualized for an optimal SOUND reconstruction of the annular plane, the cusps, and the sinus of Valsalva.

\section{SOUNDSTAR reconstruction of pulmonary valve and contiguous structures}

The Soundstar catheter is advanced in the right atrium and positioned in "home view" through a femoral vein [22]. After choosing a stable reference (i.e. surface ECG), with the ICE catheter in contact with the cardiac tissue, respiration gating ( $R G$ ) is acquired. Thus, contour acquisition can start. In order to increase the accuracy of the reconstruction and procedural safety, it is highly recommended that contours be acquired in the same cardiac (i.e. end of QRS) and respiratory phase. The steps for an optimal anatomical 
reconstruction workflow are listed below:

1. With the ICE beam in "home view" position, the contours of the right ventricle inflow and outflow tracts (with tags on tricuspid annulus), right coronary cusp (RCC), non-coronary cusp (NCC), and AORTA can be acquired (Fig. 2, Panel A). ICE images are displayed in two dimensions. Thus, in order to fill all the volume of the cardiac chambers and main vessels, multiple scans must be acquired, possibly on several planes. The more scans are acquired, the more the volume is filled in. By performing counterclockwise and clockwise rotations of the handpiece, it is possible to acquire more lateral (Fig. 2, Panel B) and septal (Fig. 2, Panel C) portions of RV and tricuspid valve, respectively. In each scan it is also recommended to acquire tags of the tricuspid annulus in order to anatomically open the tricuspid orifice. Overcoming the more septal projection of the tricuspid valve, a further clockwise rotation of the handpiece allows visualization of the most septal portion of the right ventricle outflow tract (RVOT) together with the AORTA [RCC, NCC, and left ventricle (LV)] (Fig. 2, Panel D). At this level one can appreciate the semilunar aspect of the aortic valve. Often it is possible to discern between the valvular annulus, the sinus of Valsalva and the sinus-tubular junction.

2. By taking advantage of the markers related to the tricuspid valve, which have been acquired on various projections, it is possible to highlight the valve on the SOUND MAP of the RV and open it as an "anatomical structure." This allows the operator to safely advance the ICE catheter into the right ventricle with an anterior deflection of the probe. (We suggest setting the CARTO "main window" and the "additional window" to have a RAO projection and a posterior view to help navigate the tricuspid ring). Once inside the RV, keeping the ICE beam pointing downwards, it is possible to acquire the RV apex in different positions by means of slight clockwise or counterclockwise rotations (Fig. 3, Panel A). During these acquisitions it is possible to visualize intracavitary structures, such as the moderator band and papillary muscles, reconstructing their thickness into the sound map, if necessary, for ablation purposes (not the subject of this paper). By advancing the ICE catheter to the base of the RVOT and rotating clockwise, it is possible to acquire the classic LV projections up to the mitral valve (Fig. 3, Panel B).

3. A further clockwise rotation allows the aortic valve to be visualized in short-axis view. In this projection it is possible to acquire the aortic cusps (left, right, and non-coronary). Note: the aortic valve has a semilunar shape; thus, the three cusps are also well visualized in the same scan when the left main (LM) of the left coronary arteries appears (Fig. 4, Panel A). In general, the LM arises $1 \mathrm{~cm}$ above the valvular plane and the contours of the three cusps should not be taken at this level in order to avoid designing the valvular plane at too high a level. At this stage, if the clinical practice of the imaging center allows integration with CT images, it is highly recommended to acquire FLOATING OS contours on cusp junctions and on the insertion of the LM. Once the LM has been identified, with a slight clockwise rotation it should be possible to follow and acquire the bifurcation in the anterior descending artery and circumflex artery (Fig. 4, Panel B). At this level, multiple scans can be performed to acquire each tract of these arteries in short-axis view. Sometimes it is possible to follow (and reconstruct) the anterior descending artery to the medial tract (Fig. 5, Panel A and B).

4. For both the aortic and pulmonary cusps, slight rotations are recommended in order to acquire more projections to improve the definition and correct proportions. Proceeding with clockwise rotation, passing the short axis of the aortic valve, the ICE beam cuts the most septal portion of the pulmonary artery in long-axis view. Here, it is possible to acquire the LCC, RCC, RVOT, LPC, and PA (Fig. 6, Panel A). With a slight clockwise rotation, the anterior cusp of the pulmonary valve is also displayed. This will allow reconstruction of the APC, LPC, PA, and RVOT (Fig. 6, Panel B). Depending on the anatomy of the patient, it may be necessary to slightly advance or withdraw the probe. Rotating further clockwise will give access to viewing the right cusp of the pulmonary valve (RCP) on the long axis; hence, it will be possible to acquire the RVOT, AORTA, RPC, and PA (Fig. 6, Panel C). All through the described acquisitions, it is also crucial to tag valvular points at the level of the leaflet attachments on the annulus, in order to open the valve as an "anatomical structure." During each phase of point 4, the operator must be careful to fill in as much as possible of the right ventricle volume under the valve, spanning the diameter from the septal to lateral wall: this will help to provide 
a more accurate, extended navigation of the electro-catheters (Video S1). The valvular annulus points (acquired in "floating" modality, always at the end of QRS, with the valve closed) will be kept as reference for the valve plane in the other projections (see step 6 below).

5. Once the ICE beam is directed towards the most lateral aspect of the RV wall, a gentle withdrawal of the probe to the RV inflow region (under the HIS location) sometimes allows the aorta to be displayed from below. Refining the projections with probe rotations makes it possible to recognize the origin of the right coronary artery $1 \mathrm{~cm}$ above the valve plane, at the bottom of the right sinus of Valsalva (Fig. 7, Panel A). From this point, further counterclockwise rotations make it possible to reconstruct the entire medial tract of the artery while it crosses the atrioventricular groove between the right ventricle and the right atrial appendage (Fig. 7, Panel B). These projections allow further RV volume to be filled in.

6. The final part of the workflow acquires images of the pulmonary cusps and vessel in short-axis view. The general maneuvers to perform are as follows: withdrawing the probe in the "home view," advancing the ICE catheter upwards 1-3 cm above into the atrium or towards the superior vena cava (SVC), making a slight tilt of the handpiece to the right (for a posterior deflection of the beam), and applying a clockwise rotation. The beam should cut the pulmonary artery at the valve level. The already reconstructed SOUND map can guide the operator accurately during the slight rotation and tilting movements (Video S2). This projection can display the APC, LPC, RPC, AORTA [the LPC can be found closer to the RCC; the cusp oriented to the right side (away from LV) is the RPC, while the one farthest from the aorta is the APC] (Fig. 8, Panel A). The reconstruction of the valve cusps in short-axis view may be misleading if the contours are not traced in the same ECG trace (at the end of the QRS); hence, one must keep in mind that the valve is semilunar, and, therefore, the commissures lines may also be seen $1 \mathrm{~cm}$ above the ventricular arterial junction. This is why the previous acquired valvular annulus tags (during long-axis visualization) must be used as references for the valvular plane. If the image cannot be set on the exact plane, contours of the intersections between the cusps (commissures) can be acquired as "floating" instead of as "map." It will give an idea where each cusp lies on the cranial view (Fig. 8, Panel B). At this point, slight clockwise and counterclockwise rotations can be performed in order to cross the AP and RVOT in short-axis view, filling the volume of these structures into the SOUND map (Fig. 8, Panel C).

\section{Final considerations: a tentative re-classification of ventricular arrhythmias originating from the right ventricle outflow tract.}

Once the described workflow has been completed, an accurate reconstruction of the PV plane, the PA, and the RV underneath the valve is obtained, and this allows to easily navigate the catheters in the map using multiplanar orientations [22]. Usually, we display two contemporary zoomed projections of the pulmonary valve: one on the sagittal plane (from right or left view) or on the coronal plane (from anterior or posterior view); and one on the transverse plane, from the superior (cranial) or inferior (caudal) view. In this way, using the sagittal or coronal projections, we identify if the catheter is above or below the valve. Conversely, using transverse projections, we can know with certainty which wall the catheter is in perfect contact with (the lateral, septal, anterior, medial, or posterior wall). Navigating the regions underneath or above the valve, we can be assisted by a cranial or caudal view respectively. Moving the region of interest from a lateral to a septal aspect, we can change between a right or left view.

Since we use ICE systematically, we realized the former classification of the anatomical location of RVOTtype VAs [3] can be overcome. In fact, the old historical division into three sites (site 1, site 2, and site 3) identifying the posterior, medial, and anterior aspects of the septum of the lateral wall was simply based on radioscopic views (RAO and LAO). In our opinion, a new classification, similar to that used for aortic cusp VAs, should be used. Independently if the origin of the VAs is above or below the pulmonary valve, the regions to be described should be the RPC area, the LPC area, and the APC area. According to this new concept, with rare exceptions, the RPC should comprise the old sites 1-2 of the lateral free wall, the APC should encompass the old site 3 of both the free and septal walls, and the LPC should correspond to the old sites 1-2 of the septal wall. If more accuracy is desired, one could specify if the point of interest 
is in a junction area (LPC-APC junction, RPC-LPC junction, or RPC-APC junction). This classification is not only more anatomically appropriate, it also brings the advantage of improving etiological knowledge of these forms of arrhythmias. Indeed, proving that pulmonary cusps may be the real site of origin of all RVOT-type VAs is a fascinating and elegant hypothesis to be demonstrated through high definition. For this reason, the work by Zhang et al is pioneering, but it is limited by the use of pulmonary angiography as the method for visualizing the pulmonary cusps [13]. In our experience, ICE with CARTOSOUND? map reconstruction allows a 3D reconstruction in great detail, avoiding fluoroscopy and iodine contrast use with a pigtail catheter [23]. Beside this, CARTOSOUND? module allows "in vivo" reconstruction of the course of coronary arteries, easily of the left one, whit more complexity for the right one. As mentioned above, this is relevant in term of safety of ablation, because of close proximity of left main, its bifurcation and the proximal part of left anterior descending with the left pulmonary sinus of Valsalva. Finally, SOUND maps could be merged with TAC or MRI 3D shell reconstruction of the heart (CARTOMERGE? and CARTOSEG? module) thus providing a definitely "image-guided" procedural workflow with maximized ratio between efficacy and safety [24].

\section{Acknowledgements}

The authors want to sincerely thank Nicola Zaurino and Giuliano Cerruti, competent and dedicated engineers from Biosense and Webster, who hardly worked in the images and videos reconstructions.

\section{References}

1. Ventura R, Steven D, Klemm HU, Lutomsky B, Müllerleile K, Rostock T, Servatius H, Risius T, Meinertz T, Kuck KH, Willems S. Decennial follow-up in patients with recurrent tachycardia originating from the right ventricular outflow tract: electrophysiologic characteristics and response to treatment. Eur Heart J. 2007 Oct;28(19):2338-45

2. Cronin EM, Bogun FM, Maury P, Peichl P, Chen M, Namboodiri N, Aguinaga L, Leite LR, Al-Khatip SM, Anter E, Berruezo A, Callans DJ, Chung MK, Cuculich P, d'Avila A, Deal BJ, Della Bella P, Deneke T, Dickfeld TM, Hadid C, Haqqani HM, Kay GN, Latchamsetty R, Marchlinski F, Miller JM, Nogami A, Patel AR, Pathak RK, Saenz LC, Santangeli P, Sapp JL, Sakozy A, Soejima K, Stevenson WG, Tedrow UB, Tzou WS, Varma N, Zeppenfeld K. 2019 HRS/EHRA/APHRS/LAHRS expert consensus statement on catheter ablation of ventricular arrhythmias. Heart Rhythm 2020 Jan;17(1): e155-e205.

3. Joshi S, Wilber DJ. Ablation of idiopathic right ventricular outflow tract tachycardia: current perspectives. J Cardiovasc Electrophysiol 2005;16: S52-58.

4. Dixit S, Gerstenfeld EP, Callans DJ, Marchlinski FE. Electrocardiographic patterns of superior right ventricular outflow tract tachycardias: distinguishing septal and free-wall sites of origin. J Cardiovasc Electrophysiol. 2003; 14:1-7.

5. Timmermans C, Rodriguez LM, Medeiros A, Crijns HJGM, Wellens HJJ. Radiofrequency catheter ablation of idiopathic ventricular tachycardia originating in the main stem of the pulmonary artery. J Cardiovasc Electrophysiol 2002; 13: 281-4;

6. Timmermans C, Rodriguez LM, Crijns HJGM, Moorman A, Wellens HJJ. Idiopathic left bundlebranch block- shaped ventricular tachycardia may originate above the pulmonary valve. Circulation 2003; 108:1960-7.

7. Sekiguchi Y, Aonuma K, Takahashi A, Yamauchi Y, Hachiya H, Yokoyama Y, Iesaka Y, Isobe M. Electrocardiographic and Electrophysiologic Characteristics of Ventricular Tachycardia Originating Within the Pulmonary Artery. J Am Coll Cardiol 2005; 45:887-95

8. Suleiman M, Asirvatham SJ. Ablation above the semilunar valves: When, why, and how? Part I. Heart Rhythm 2008;5: 1485-1492

9. Liao Z,Zhan X, Wu S, Xue Y, Fang X, Liao H, Deng H, Liang Y, Wei W, Liu Y, Ouyang F Idiopathic Ventricular Arrhythmias Originating From the Pulmonary Sinus Cusp. Prevalence, Electrocardiographic/Electrophysiological Characteristics, and Catheter Ablation. J Am Coll Cardiol 2015; 66:2633-44

10. Liang Z, Ren X, Zhang T,Jianzeng Dong ZH, Wang Y Mapping and ablation of RVOT-type arrhyth- 
mias: comparison between the conventional and reversed U curve methods. Journal of Interventional Cardiac Electrophysiology (2018) 52:19-30

11. Yang Y, Liu Q, Liu Z, Zhou S. Treatment of pulmonary sinus cusp-derived ventricular arrhythmia with reversed U-curve catheter ablation. J cardiovasc electrophysiol 2017;28(7):768-775

12. Insights on the pulmonary artery-derived ventricular arrhythmia. J Cardiovasc Electrophysiol 2018;29(9):1330-1337

13. Zhang J, Tang C, Zhang Y, Su X. Pulmonary Sinus Cusps Mapping and Ablation: A New Concept and Approach for Idiopathic Right Ventricular Outflow Tract Arrhythmias. Heart Rhythm 2018 Jan;15(1):38-45

14. Anderson RH, Mohun TJ, Sanchez-Quintana D, Mori S, Spicer DE, Cheung JW, Lerman BB. The anatomic substrates for outflow tract arrhythmias. Heart Rhythm 2019; 16:290-297

15. Gami AS, Noheria A, Lachman N, Edwards WD, Friedman PA, Talreja D, Hammill SC, Munger TM, Packer DL, Asirvatham SJ. Anatomical correlates relevant to ablation above the semilunar valves for the electrophysiologist: a study of 603 hearts. J Interv Card Electrophysiol 2011 Jan;30(1):5-15

16. Liu CF, Cheung JW, Thomas G, Ip JE, Markowitz SM, Lerman BB. Ubiquitous Myocardial Extensions Into the Pulmonary Artery Demonstrated by Integrated Intracardiac Echocardiography and Electroanatomic Mapping Changing the Paradigm of Idiopathic Right Ventricular Outflow Tract Arrhythmias. Circ Arrhythm Electrophysiol 2014; 7:691-700

17. Stamm CG, Anderson RH, Ho SY. Clinical anatomy of the normal pulmonary root compared with that in isolated pulmonary valvular stenosis. J Am Coll Cardiol 1998; 31:1420-1425

18. Walsh KA, Fahy GJ. Anatomy of the left main coronary artery of particular relevance to ablation of left atrial and outflow tract arrhythmias. Heart Rhythm 2014;11(12):2231-2238

19. Futyma P, Santangeli P, Purerfellner H, Pothineni NV, Gluszczyk R, Ciapala K, Moroka K, Martinek M, Futyma M, Marchilinski FE, Kulakowski P. Anatomic approach with bipolar ablation between the left pumonic cusp and left ventricular outflow tract for left ventricular summit arrhythmias. Heart Rhythm 2020; 26:S1547-5271

20. Dong X, Tang M, Sun Q, Zhang S. Anatomical relevance of ablation to the pulmonary artery root: Clinical implications for characterizing the pulmonary sinus of Valsalva and coronary artery. J Cardiovasc Electrophysiol 2018; 29(9):1230-1237

21. Ehdaie A, Liu F, Cingolani E, Wang X, Chugh SS, Shehata M. How to use intracardiac echocardiography to guide catheter ablation of outflow tract ventricular arrhythmias. Heart Rhythm 2020 Mar 6; S1547-5271

22. Enriquez A, Saenz LC, Rosso R, Silvestry FE, Callans D, Marchlinski FE, Garcia F. Use of intracardiac Echocardiography in Interventional Cardiology. Working With the Anatomy Rather Than Fighting it. Circulation. 2018; 137:2278-2294

23. De Sensi F, Addonisio L, Limbruno U. Premature ventricular complexes ablation above the anterior pulmonary cusp with reversed U curve under intracardiac echocardiography guidance. J Interv Card Electrophysiol. 2020 Jan;57(1):105-106

24. De Sensi F, Miracapillo G, Cresti A, Paneni F, Limbruno U. Image integration guided ablation of left outflow tract ventricular tachycardia: Is coronary angiography still necessary? Indian Pacing Electrophysiol J 2018;18(2):73-75

\section{Figure Legends}

\section{Figure 1}

\section{Computed tomography images of the heart .}

Panel $A$. axial scan: from a caudal view the reader can recognize the PV as the most anterior (in the superior aspect of the image) structure of the heart in close contact with the basal septum and aortic root. The space between LPC, LCC and LVOT is the LV summit. While the PV is cut "on face", the AV is cut "on edge". Panel B : sagittal scan: from a left side view is clearly appreciable the closeness between LPC and Left Main coronary artery. In this projection the PV is cut "on edge", while the AV is cut "on face". 
The PV lies at a level just superior to the AV and runs on an oblique plan (from antero-superior towards postero-inferior position). LV: left ventricle, RV: right ventricle, $\mathrm{AV}$ : aortic valve, $\mathrm{PV}$ : pulmonary valve, APC: anterior pulmonary cusp, RPC: right pulmonary cusp, LPC: left pulmonary cusp, LCC: left coronary cusp, RCC: right coronary cusp, NCC: non coronary cusp, LVOT: left ventricle outflow tract, RVOT: right ventricle outflow tract, RA: right atrium, RAA: right atrial appendage, LA: left atrium, LAA: left atrial appendage, LM: left main

\section{Figure 2}

Step 1: initial reconstruction of the right ventricle and aortic root. (2D intracardiac echo frames with the ICE beam visualized are fused with CARTOSOUND? reconstruction of some structures for the reader to better understand the 3D anatomy and the relationship between right and left structures).

Panel A : ICE catheter in "home view" neutral position. Acquisition of TV tags, RV volume, AV in long axis view with visualization of RCC and NCC. Panel B: ICE catheter rotated counterclockwise (anterior) to acquire a more lateral portion of the right ventricle (RV). Acquisition of other TV tags and other RV contours. Panel C: ICE catheter rotated clockwise (posterior) to acquire a more septal portion of the right ventricle (RV). Acquisition of other TV tags and other RV contours. Delineation of aortic valvular plane. Panel D: ICE catheter rotated more clockwise to acquire the most septal portion of the right ventricle outflow tract (RVOT). The aorta with aortic cusps (NCC and RCC) and left ventricle (LV) (at the level of the outflow tract) are also visible on the same projection. ICE: Intracardiac echocardiography, TV: tricuspid valve, RV: right ventricle, AV: aortic valve, RCC: right coronary cusp, NCC: non coronary cusp, LCC: left coronary cusp, PA: pulmonary artery.

Figure 3

\section{Step 2: detailed reconstruction of right ventricle and septum.}

Panel A: Exploiting the TV tags, with anterior flection the ICE catheter is advanced into the RV at basal level with the beam facing downward. With gentle movements of the probe other RV contours are acquired to fill the RV volume. It is possible at this level to distinguish intracavitary structures (i.e. papillary muscles, moderator band). Panel B: With serial clockwise rotations LV is displayed and reconstructed with the possibility to reconstruct also the papillary anatomy (not the aim of this paper). TV: tricuspid valve, RV: right ventricle, LV: left ventricle, PA: pulmonary artery.

\section{Figure 4}

\section{Step 3: reconstruction of aortic valve and left coronary artery.}

Panel A: With the ICE catheter positioned at the base of the RVOT with the beam facing upwards the $\mathrm{AV}$ is displayed in short-axis view. Aortic cusps (LCC, RCC, NCC) can be reconstructed. When the LM is visualized aortic cusps contours should not be traced (the beam is already cutting at the level of the sinus of Valsalva). Panel B:From the same position of Panel A, a slight counterclockwise rotation allows the operator to follow the LM until the bifurcation in LAD and LCX. Multiple contours of these vessels traced in short axis view (with gentle clockwise and counterclockwise torsions) permit the SOUND reconstruction of the coronaries. ICE: intracardiac echocardiography, RVOT: right ventricle outflow tract, AV: aortic valve, LCC: left coronary cusp, RCC: right coronary cusp, NCC: noncoronary cusp, LM: left main, LAD: left anterior descendent (artery), LCX: circumflex (artery).

Figure 5

\section{Step 3: delineation of the left anterior descendent artery course.}

Panel $A$ : The ICE probe is positioned at the base of the RVOT with the beam directed upwards visualizing the space between the two semilunar valves and arterial roots. This is the tract where the LAD is closest to the PA. Only LPC is displayed. Panel B : with slight counterclockwise rotations it is possible to follow the 
entire course of the LAD until its medial (exceptionally the distal) tract evaluating its proximity with the LPC. ICE: intracardiac echocardiography, RVOT: right ventricle outflow tract, LAD: left anterior descendent (artery), PA: pulmonary artery, LPC: left pulmonary cusp.

\section{Figure 6}

\section{Step 4: visualization and reconstruction of the pulmonary valve in long-axis view.}

Panel A: ICE catheter at the base of the right ventricle outflow tract (RVOT) with the beam oriented upwards. The most septal portion of PV in long axis view is visualized. At this level part of the RVOT, LPC, PA, RCC and LCC are displayed. Panel B: a gentle clockwise rotation allows to visualize both LPC and APC. Panel $C$ : with further clockwise torsion the beam cuts the right pulmonary cusp "on edge, the visualization of the aorta indicates a posterior direction of the beam. During each phase of this step annular tags must be acquired.

\section{Figure 7}

\section{Step 5: visualization and reconstruction of the right coronary artery.}

Panel A: ICE catheter is withdrawal under the HIS location with the US beam directed upwards. Aorta and PA are both visualized in the long axis view; sometimes, at this, level the origin of right coronary artery can be traced. Panel B: slight clockwise rotations allow to follow and reconstruct the proximal course of the R_CORO in the AV groove. Further RV volume can be filled in. ICE: intracardiac echocardiography, US: ultrasound, PA: pulmonary artery, AV: atrioventricular, RV: right ventricle.

\section{Figure 8}

\section{Step 6: visualization and reconstruction of the pulmonary valve in short-axis view.}

Panel A: from the "home view," gently advancing the probe towards the SVC, while slightly tilting posteriorly and towards right and by making a clockwise or counterclockwise rotation (depending on the anatomy), the operator should be able to orient the beam on the pulmonary artery. The aortic valve is displayed in long-axis view.Panel $B$ : intracardiac echocardiography (ICE) catheter in the right atrium with the beam oriented on the short axis of the pulmonary artery.

\section{Supplemental material}

\section{Video S1}

Visualization of the pulmonary valve in the long axis view from the base of the RVOT. Contours tracings and reconstruction in the CARTOSOUND? map.

\section{Video S2}

Visualization of the pulmonary valve in the short axis view from the right atrium /SVC. 

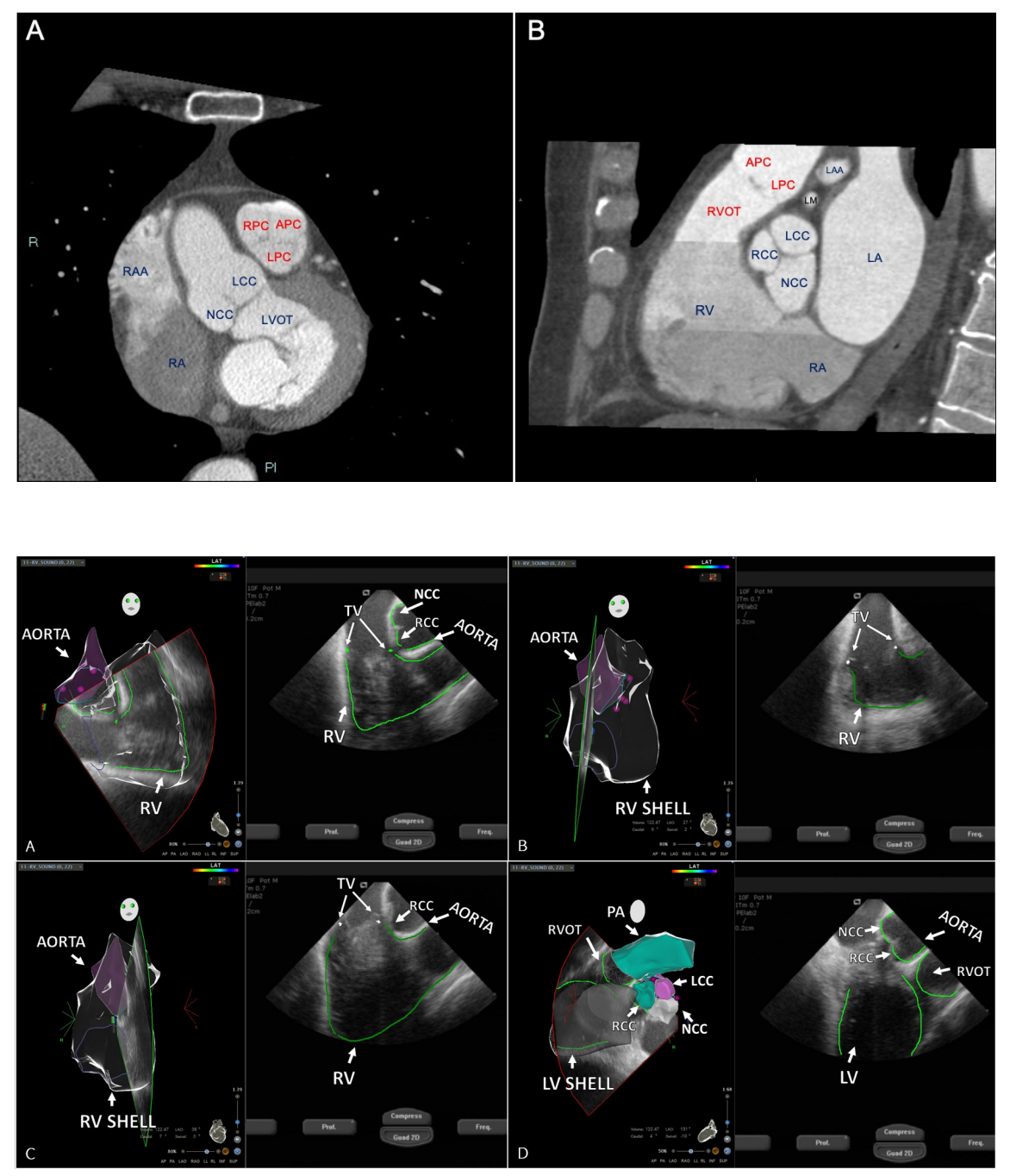


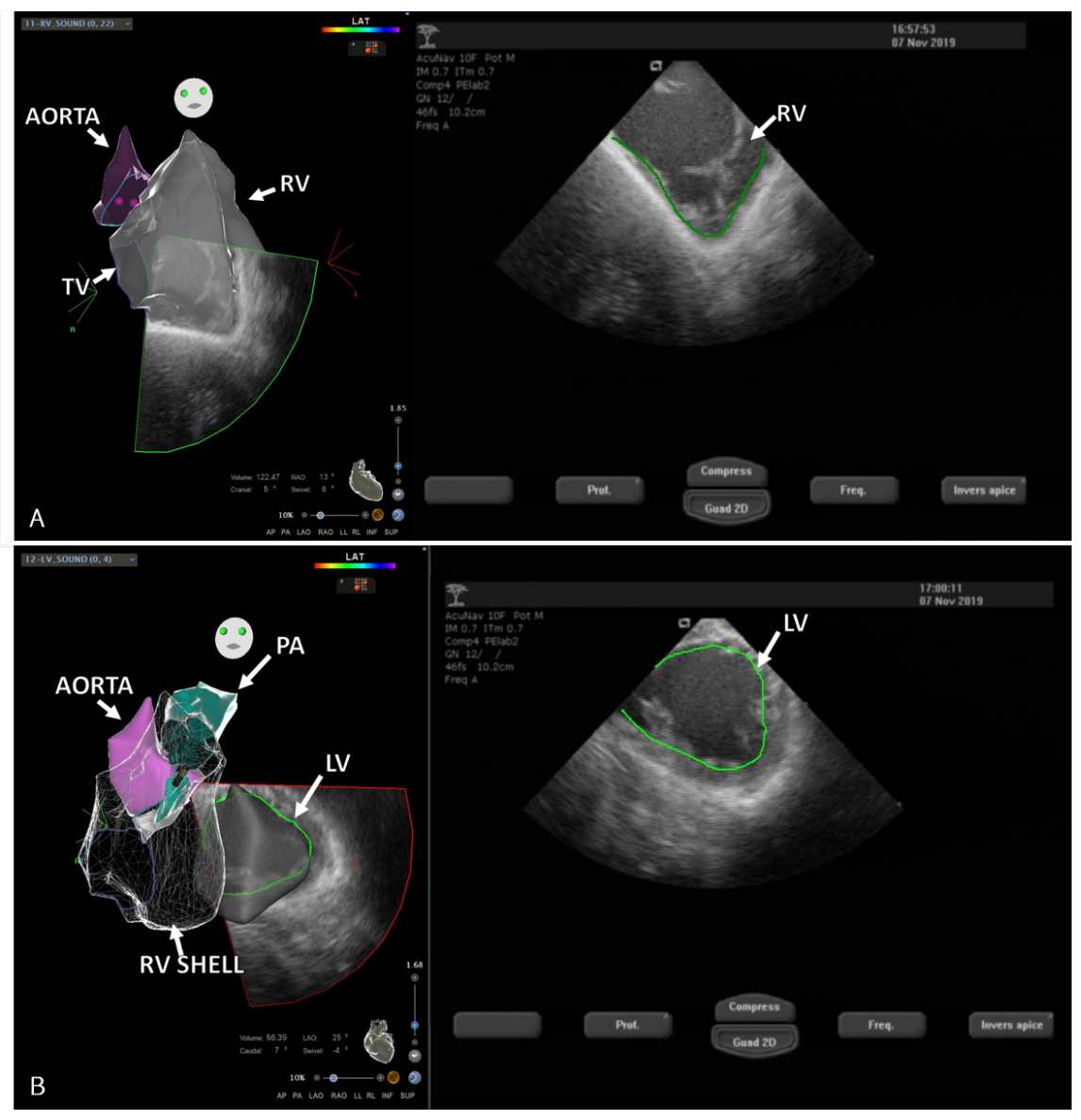




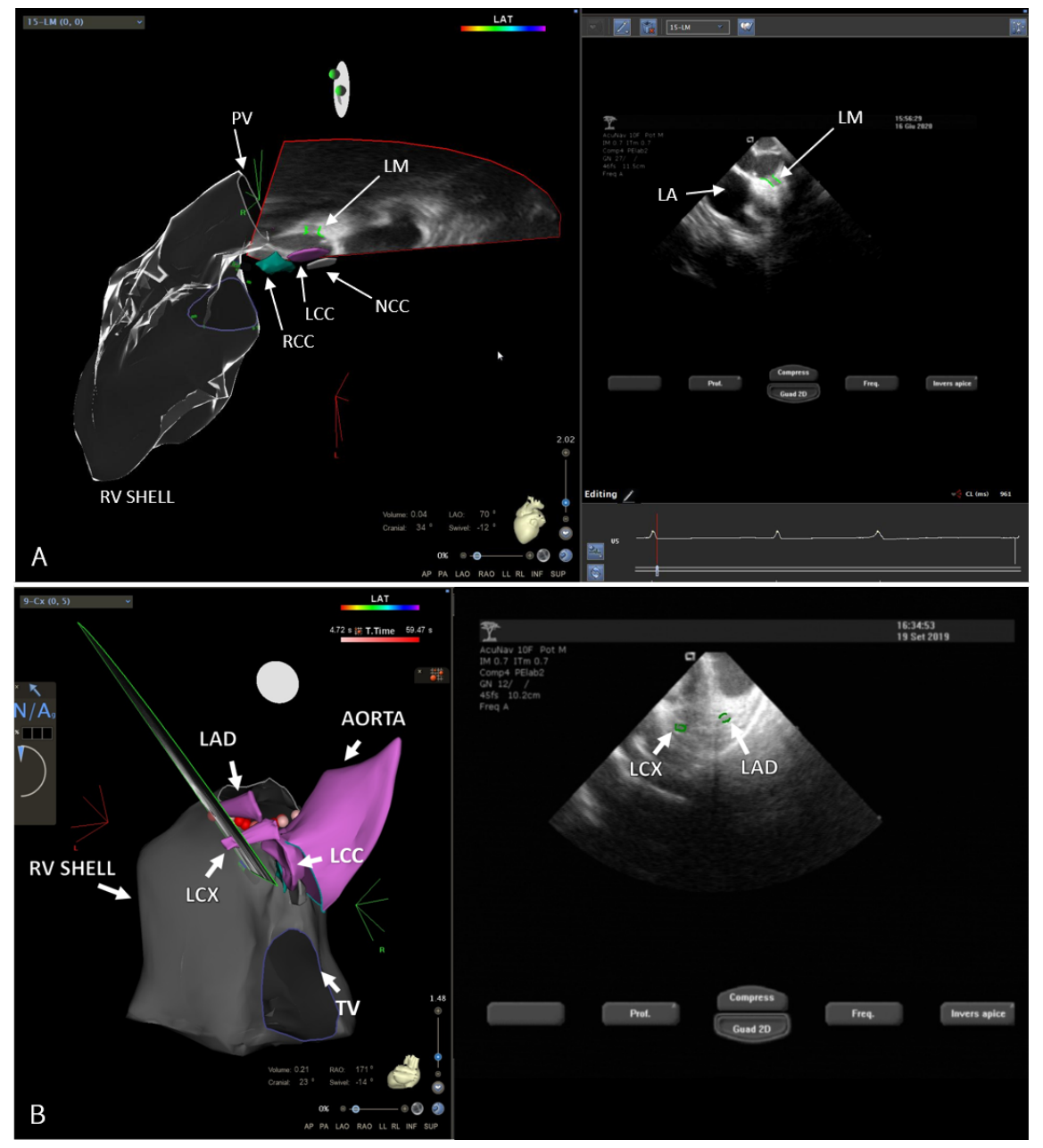




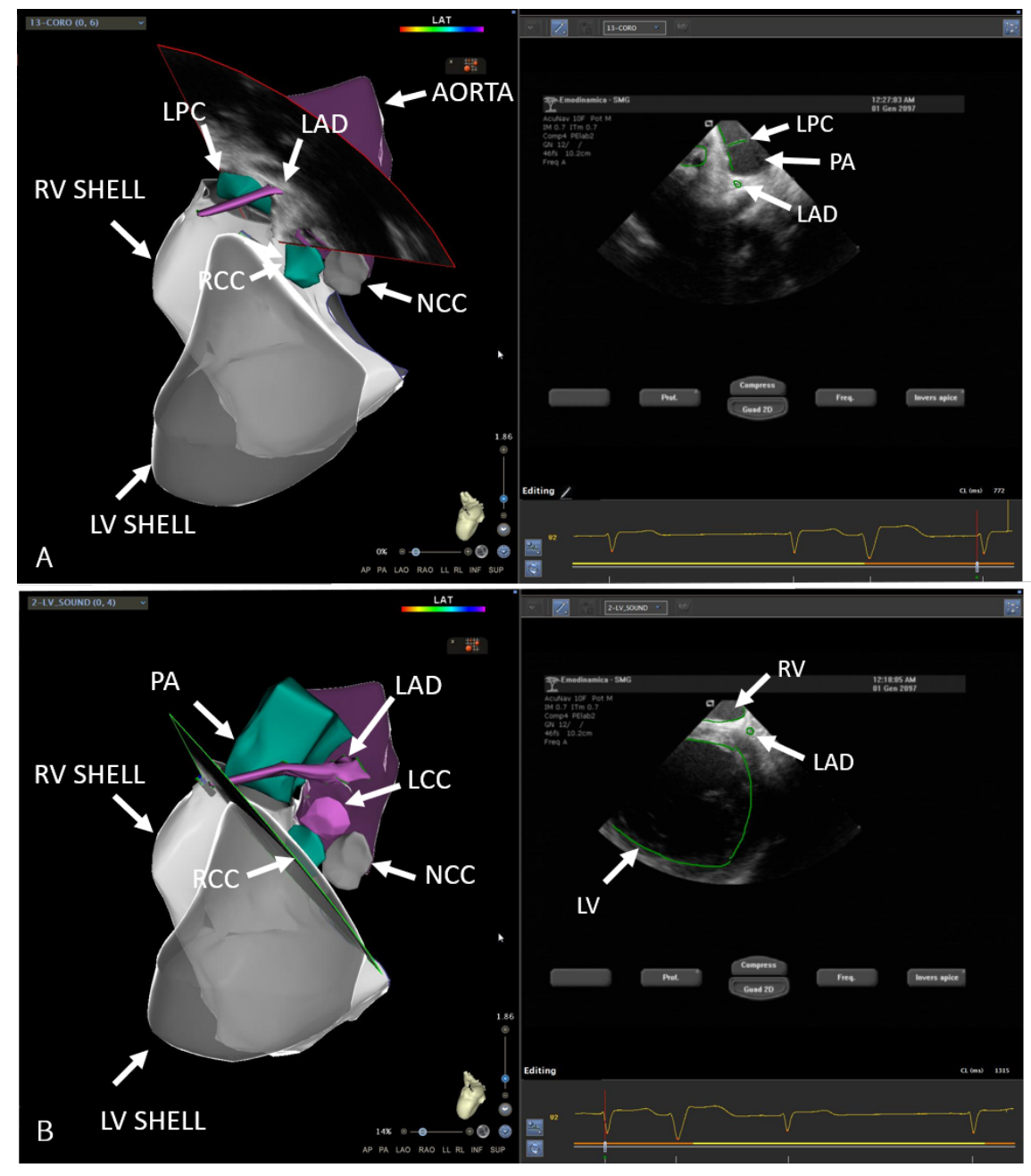




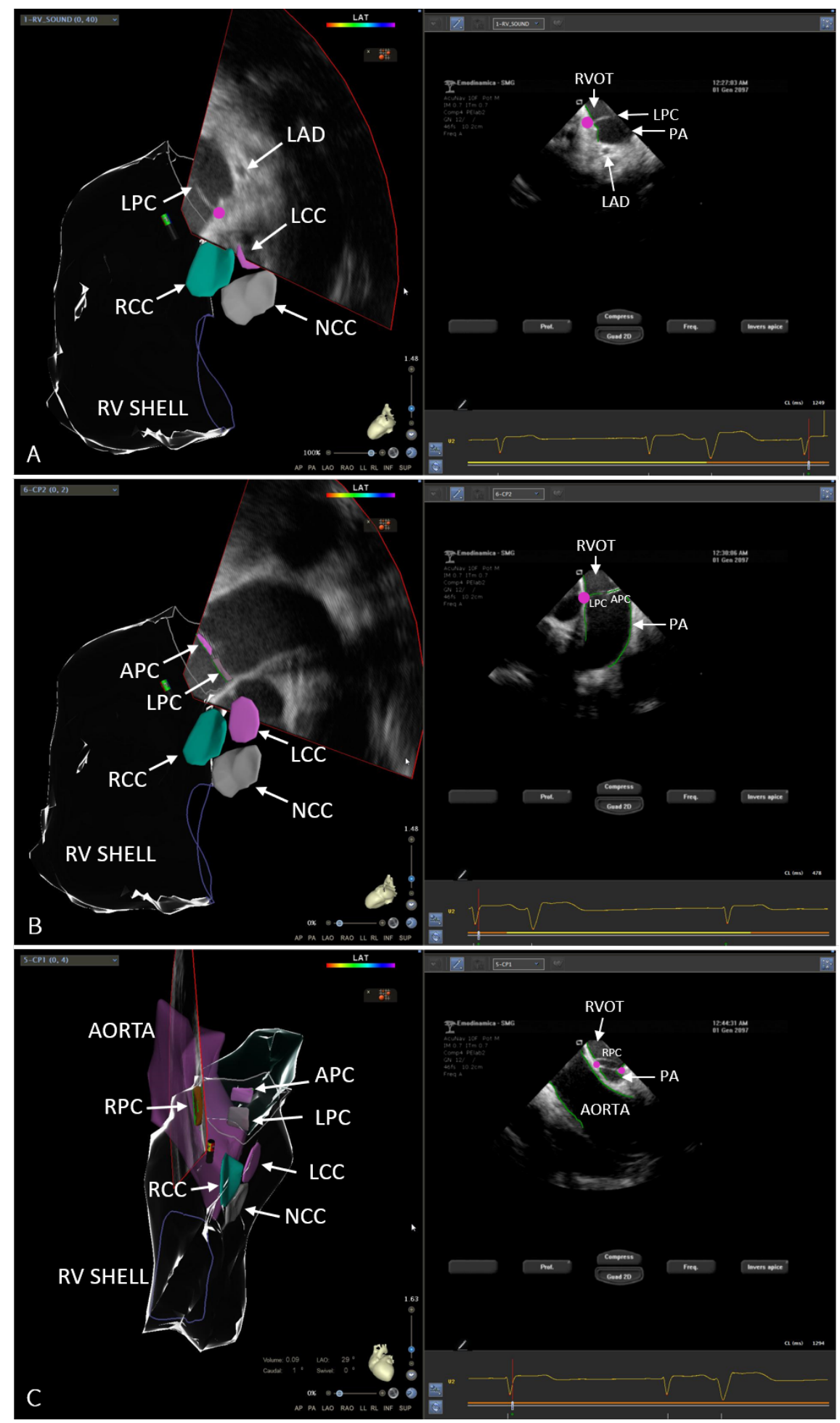




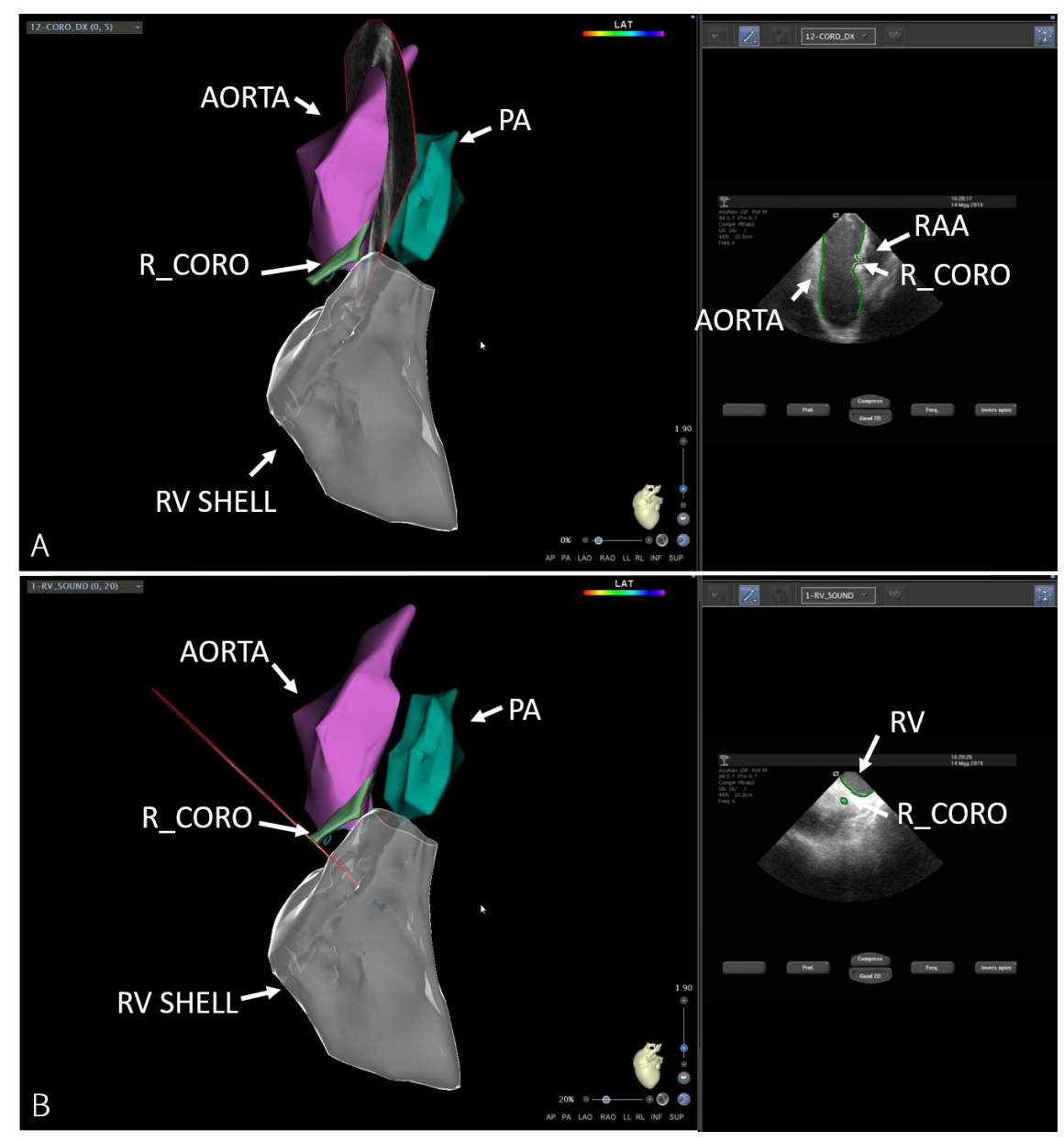




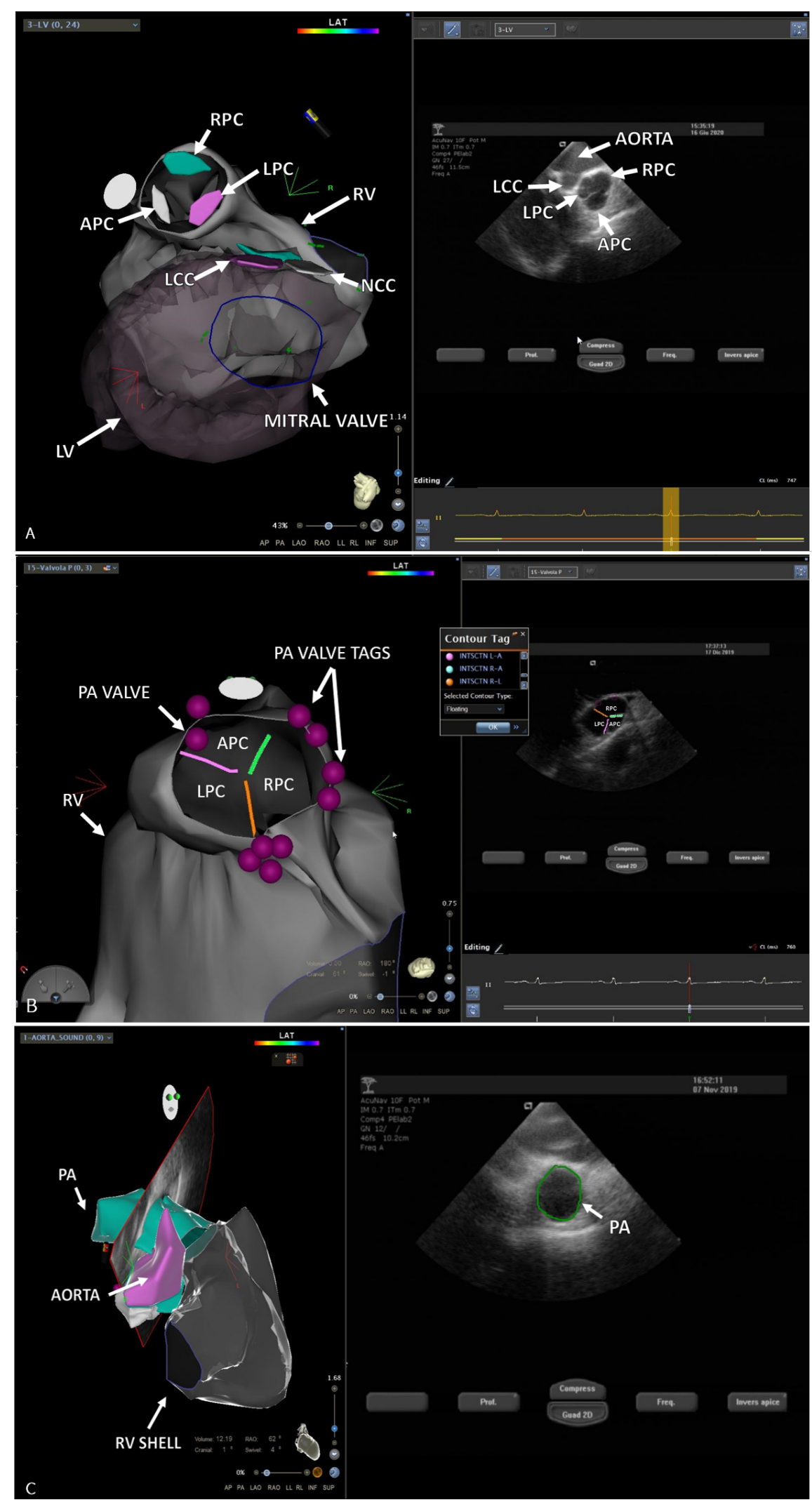

